hep-th/9705188, PUPT-1680

\title{
Higher Loop Effects in M(atrix) Orbifolds.
}

\author{
Ori J. Ganor, Rajesh Gopakumar and Sanjaye Ramgoolam \\ Department of Physics, Jadwin Hall \\ Princeton University \\ Princeton, NJ 08544, USA \\ origa, rgk, ramgoola@puhep1 . princeton .edu
}

\begin{abstract}
Scattering of zero branes off the fixed point in $R^{8} / Z_{2}$, as described by a super-quantum mechanics with eight supercharges, displays some novel effects relevant to Matrix theory in non-compact backgrounds. The leading long distance behaviour of the moduli space metric receives no correction at one loop in Matrix theory, but does receive a correction at two loops. There are no contributions at higher loops. We explicitly calculate the two-loop term, finding a non-zero result. We find a discrepancy with M(atrix)-theory. Although the result has the right dependence on $v$ and $b$ for the scattering of zero branes off the fixed point the factors of $N$ do not match. We also discuss scattering in the orbifolds, $R^{5} / Z_{2}$ and $R^{9} / Z_{2}$ where we find the predicted fractional charges.
\end{abstract}

May, 1997 


\section{Introduction}

The boldness of the proposal for an exact formulation for 11D M-theory [1] has provoked a rather intensive comparison of this theory with known limits of M-theory. Many studies have been undertaken of simple compactifications and properties of BPS states therein. [1, 2, 3, 4, 4, 5, 6, 17, 8, 9, 10, 11, 12, 13, 14, 15, 16, 17] The type-IIA string theory limit [18, 19,20] together with interactions [20] has been argued to arise naturally. The perturbative heterotic theory and its compactifications have also been recently studied [21,22, 23,24].

Another line that has been pursued is the traditional one of scattering objects (mostly BPS) off each other. It was one of the interesting features of the original proposal that the "tree-level" $\frac{v^{4}}{b^{7}}$ effective potential between gravitons in M-theory was reproduced by a 1-loop effect in the 0+1D SYM [ [] . The leading small velocity and long distance behaviour in the scattering of many brane configurations are also reproduced exactly by 1-loop calculations [25,26,27]. An interesting calculation for a process with non-zero longitudinal momentum transfer has also been carried out in [28] and yields the correct result.

One motivation for the present paper was to study the loop expansion of M(atrix)theory. It is natural to look for effects in $\mathrm{M}$ (atrix)-theory that can only be seen at higher loops in the Quantum Mechanics. This could be particularly educative in cases with less than maximal supersymmetry. As we will see, such a situation in which we can see a qualitative effect only by going to two loops, arises in the scattering off the fixed point of $\mathbb{R}^{8} / \mathbb{Z}_{2}$. In M-theory language, what we are probing is a quantum effect - the effective fractional membrane charge at the fixed point which is related to the tadpole discovered in [29]. In principle, the details of the bound-state in the large- $N$ limit could contribute as well. Its details are mostly unknown and in fact, it is only recently, that the existence of an $S U(2)$ bound state has been proven rigorously [30]. We will show that the details of the bound state wave-function will contribute only at subleading order.

A parallel motive for this work was to understand something about the crucial issue of compactifications on nontrivial backgrounds. The prescription given in [1] for toroidal compactification was to use "large" gauge transformations of the model. We recall that when $N=\infty$ states have to be invariant under "small" gauge transformations, i.e. $U \in$ $U(\infty)$ such that $\|U-\mathbf{I}\|<\infty$. By using appropriate "large" gauge transformations (i.e. $U \in U(\infty)$ such that $\|U-\mathbf{I}\|=\infty$ ) one can map toroidal compactifications to SYM theories in various dimensions [1,2,20, 4 . Nontoroidal compactifications have been studied in [31,32.

There seem to be several problems with such compactifications. In general, compactifications to less that eight dimensions seem to contain a gauge theory in more than $3+1 \mathrm{D}$ 
and those are not renormalizable (see [18] for a discussion on this subject). In compactifications to lower dimensions it seems necessary to use the newly discovered [33] chiral theories in 5+1D 11, 16, 34 but these do not seem to appear naturally from matrices.

Therefore, we do not have at the moment a prescription for how to formulate $\mathrm{M}$ (atrix) theory on a general background. The orbifold limits of compactifications have been formulated according to the prescription of [1] by restricting to the part of the parameter space that is invariant under "large" gauge transformations. The authors of [32] have pointed out several problems with this model in deforming away from the orbifold point and have shown that compactifications on K3 cannot be reproduced by a finite number of "off-diagonal" degrees of freedom.

In order to "isolate" the problems, it seems worthwhile to study the non-compact orbifolds $\mathbb{R}^{d} / \Gamma$. These would be given by a $0+1 \mathrm{D}$ theory with less than 16 supercharges. The examples that we study have (excepting one) 8 supercharges. General theories with 8 supersymmetries in $0+1 \mathrm{D}$ have been recently studied in 35] and indeed, supersymmetry allows for higher loop corrections to the metric.1

Our paper is organized as follows. Section (2) is a review of effects near orbifolds in M-theory. We review the M-theory quantum effects near $\mathbb{R}^{4} / \mathbb{Z}_{2}, \mathbb{R}^{5} / \mathbb{Z}_{2}, \mathbb{R}^{8} / \mathbb{Z}_{2}$ and $\mathbb{R}^{9} / \mathbb{Z}_{2}$. We calculate the scattering off $\mathbb{R}^{5} / \mathbb{Z}_{2}$ and $\mathbb{R}^{9} / \mathbb{Z}_{2}$ in $\mathrm{M}$ (atrix)-theory and briefly discuss the scattering on orbifolds which accommodate non-trivial low-energy theories: $\mathbb{R}^{4} / \mathbb{Z}_{2}$ (free in the IR) and $\mathbb{R}^{6} / \mathbb{Z}_{3}$ (interacting). (Such theories have been extensively discussed in [36] from a different perspective.) In section (3) we explain why we expect the two-loop diagrams to be the leading contribution in the $\mathbb{R}^{8} / \mathbb{Z}_{2}$ case. We discuss the general behaviour of multi-loop diagrams. Our discussion implies, for example, that in the $S U(N)$ quantum mechanics at finite $N$, higher loops do not affect the $\frac{v^{4}}{r^{7}}$ term in the effective potential. In section (4) we list the technical details of the two-loop calculation near $\mathbb{R}^{8} / \mathbb{Z}_{2}$. In section (5) we look at the wave-function contribution and argue that the leading term is "universal" in the sense that it can be determined without a detailed knowledge of the bound-state wave-function. In section (6) we present the final result and in section (7) we discuss the large $N$ behaviour.

\section{Note added}

As this work neared completion, a paper which uses similar two-loop techniques in a related context appeared [37.

We have also learnt about another work 38,39 which has calculated scattering off $\mathbb{R}^{5} / \mathbb{Z}_{2}$ and $\mathbb{R}^{9} / \mathbb{Z}_{2}$. We are grateful to the author for discussions.

1 We are grateful to N. Seiberg for bringing this to our attention. 


\section{Quantum effects at orbifolds}

In M-theory there are quantum effects at various orbifolds. For example, The $\mathbb{R}^{5} / \mathbb{Z}_{2}^{\prime}$ orbifold [40,41] (the $\mathbb{Z}_{2}^{\prime}$ includes the $A_{3} \rightarrow-A_{3}$ transformation) is required to have $-\frac{1}{2}$ a 5 -brane charge at the fixed point [41]. This means that at large distances the low-energy gravitational fields are those that would be induced around an object with the mass and charge of $\frac{1}{2}$ an anti 5-brane. Similarly, at the $\mathbb{R}^{8} / \mathbb{Z}_{2}$ fixed point there is, effectively, $-\frac{1}{16}$ of a membrane charge. There are other orbifolds in which the low-energy description requires a non-trivial theory at the fixed points. For $\mathbb{R}^{4} / \mathbb{Z}_{2}$ this is the $S U(2)$ gauge theory at an $A_{1}$ singularity [42], which is non-trivial but free in the $\mathrm{IR} . \mathbb{R}^{9} / \mathbb{Z}_{2}^{\prime}$ has a $1+1 \mathrm{D}$ CFT [43]. After compactification on $\mathbf{S}^{1}$ we find as a result $-\frac{1}{32}$ of an anomalous momentum (0brane charge) 43. There are more complicated examples, like $\mathbb{R}^{6} / \mathbb{Z}_{3}$ where the singularity involves a collapse of a $\mathbf{P}^{2}$ and a non-trivial 5D IR fixed point [44].

These effects influence the scattering of gravitons off the fixed points and we are going to discuss the calculation both in the low-energy supergravity and in the quantum mechanics. The approximation that is made here is that of small momenta for the graviton in the orbifolded dimensions.

In this section we calculate the scattering off $\mathbb{R}^{5} / \mathbb{Z}_{2}^{\prime}$ and $\mathbb{R}^{9} / \mathbb{Z}_{2}$ which involve a 1 loop effect in the gauge quantum mechanics. In the next sections we will calculate the scattering off $\mathbb{R}^{8} / \mathbb{Z}_{2}$ which exhibits interesting 2-loop effects.

\subsection{Scattering off $\mathbb{R}^{5} / \mathbb{Z}_{2}^{\prime}$}

The $\mathrm{M}$ (atrix)-model for $\mathbb{R}^{5} / \mathbb{Z}_{2}$ has been given in [7, 45$]$.

The $0+1 \mathrm{D}$ Lagrangian has an $S p(N)$ gauge group. There is one gauge multiplet which contains the gauge field $A_{0}$, its 5 bosonic superpartners $X_{\mu}(\mu=1 \ldots 5)$ and 8 fermionic "gluino" superpartners $\theta_{\alpha i}$ with $\alpha=1 \ldots 4$ a spinor index of the transverse $S O(5)$ and $i=1,2$. The fields $\left(A_{0}, X_{\mu}, \theta_{\alpha i}\right)$ are in the adjoint $\mathbf{N}(\mathbf{2} \mathbf{N}+\mathbf{1})$ of $S p(N)$. The other multiplet contains 4 complex bosonic and 4 complex fermionic fields $\left(\Phi_{a}, \bar{\Phi}^{a}, \psi_{\alpha}\right)$ $(a=6 \ldots 9)$ where $\psi_{\alpha}(\alpha=1 \ldots 4)$ is a spinor of $S O(5)_{(1 \ldots 5)}$. They are in the antisymmetric $\mathbf{N}(\mathbf{2 N}-\mathbf{1})$ of $S p(N)$ and correspond to the $6 \ldots 9$ space-time coordinates.

Using similar techniques as in [46] [26] we find the phase shift for scattering one D0brane off the fixed point at impact parameter $b$, velocity $v$. The one loop determinantal contribution is:

$$
\begin{aligned}
& \operatorname{det}^{-2}\left(-\partial_{\tau}{ }^{2}+\gamma^{2} \tau^{2}+b^{2}\right) \operatorname{det}^{-1}\left(-\partial_{\tau}{ }^{2}+\gamma^{2} \tau^{2}+b^{2}+2 \gamma\right) \\
& \operatorname{det}^{-1}\left(-\partial_{\tau}{ }^{2}+\gamma^{2} \tau^{2}+b^{2}-2 \gamma\right) \operatorname{det}^{4}\left(\begin{array}{cc}
\partial_{\tau} & \gamma \tau-i b \\
\gamma \tau+i b & \partial_{\tau}
\end{array}\right) .
\end{aligned}
$$


where $\tau=i t$ and $\gamma=-i v$. Note that we have 4 transverse bosons instead of 8 for $\mathcal{N}=4$ and 8 fermions instead of 16 .

In the limit $v / b^{2} \rightarrow 0$ one finds on taking the logarithm, that the phase shift is

$$
\delta(b, v)=-\frac{v}{2 b^{2}}
$$

Remembering that the fixed point charge in the covering space (where we have been working) is twice that of the orbifold, this phase shift is in agreement 46] with half a 5-brane at the orientifold point. Thus, at 1-loop level M(atrix)-theory gives the same result, since in the type-IIA diagrams, the contribution of full SUSY multiplets cancels 47] [1].

\subsection{Scattering off $\mathbb{R}^{9} / \mathbb{Z}_{2}$}

The next example that we will discuss is M-theory on $\mathbb{R}^{1,1} \times\left(\mathbb{R}^{9} / \mathbb{Z}_{2}^{\prime}\right)$. This is a more complicated effect because of the nontrivial CFT that lives on the fixed point. There is only one homogeneous space dimension and the anomalous charge that is associated with the orbifold is a fractional unit of momentum which appears whenever we compactify the homogeneous direction on a large $\mathbf{S}^{1}$ (and disappears in the infinite radius limit). This is the signature of a nontrivial $1+1 \mathrm{D}$ CFT. The fraction of momentum is determined to be $-\frac{1}{32}$ 43.

The M(atrix) model for M-theory on $\mathbb{R}^{9} / \mathbb{Z}_{2}^{\prime}$ can be constructed as in []]. It is the dimensional reduction to $0+1 \mathrm{D}$ of $S O(2 N)$ Yang-Mills in $10 \mathrm{D}$. This model has the correct moduli space of $\left(\mathbb{R}^{9} / \mathbb{Z}_{2}\right)^{N} / S_{N}$ but at first sight this twice as many supersymmetries. However, the nonlinearly realized supersymmetries of [1], i.e.

$$
\delta \theta=\epsilon \mathbf{I}
$$

are no longer there because $S O(2 N)$ doesn't have a $U(1)$ center like $U(N)$.

We will recover the $-\frac{1}{32}$ quantum charge only in the type-IIA limit. We scatter one D0-brane off the orientifold point, as before at impact parameter $b$ and velocity $v$. The model is $S O(2)$ SYM and so there are no interactions. On the other hand the D0-brane interacts with its image and gives a force (gradient of the potential) of:

$$
F_{1}(b)=7 \frac{v^{4}}{(2 b)^{8}}
$$

since $2 b$ is the distance to the image brane. This has to be canceled by an effective D0-brane charge $Q$ ( $2 Q$ in the covering space), at the fixed point which contributes

$$
F_{2}(b)=2 Q \times 7 \frac{\left(\frac{1}{2} v\right)^{4}}{b^{8}}
$$


note that the relative velocity is $\frac{1}{2} v$ since the orientifold is fixed. Requiring the two contributions to cancel gives us precisely $Q=-\frac{1}{32}$.

In the M-theory limit, when the size of the 11th dimension is much larger that the impact parameter $b$, a graviton doesn't feel a force from the fixed point, only from its image. This agrees with $\mathrm{M}$ (atrix)-theory since in the large $N$ limit the potential between the D0-brane and its image behaves like $N^{2}$ whereas the potential between the D0-brane and fixed point behaves only like $N$.

\subsection{Scattering off a membrane in 11D supergravity}

Our main focus in this paper is the scattering off $\mathbb{R}^{8} / \mathbb{Z}_{2}$ which we will describe in detail in later sections. In this subsection we will simply calculate the expected classical supergravity result for the scattering phase shift off a membrane. We do this to leading order in $v$ and $\frac{1}{b}$. We write down the classical solution of a membrane in $10+1 \mathrm{D}$, see for example 48]:

$$
\begin{aligned}
d s^{2} & =H^{-2 / 3}\left(-d t^{2}+d y_{1}^{2}+d y_{11}^{2}\right)+H^{1 / 3} d x_{i} d x^{i}, \\
C_{3} & =H^{-1} d t \wedge d y_{1} \wedge d y_{11}, \\
H & =1+\frac{Q_{2}}{r^{6}}
\end{aligned}
$$

where $Q_{2}=8 N_{2}(2 \pi)^{2}\left(l_{p}\right)^{6}$ and $N_{2}$ is an integer. Using

$$
|\vec{p}| \frac{d p^{i}}{d t}=\Gamma_{00}^{i}|\vec{p}|^{2}+\Gamma_{j j}^{i}\left(p^{j}\right)^{2}
$$

the geodesic equation for a graviton in this background is:

$$
|\vec{p}| \frac{d p_{3}}{d t}=\Gamma_{300}|\vec{p}|^{2}+\Gamma_{333}\left(p^{3}\right)^{3}+\Gamma_{3,11,11}\left(p^{11}\right)^{2}
$$

with

$$
p_{3}=\frac{N_{0} v}{R_{11}}, \quad p_{11}=\frac{N_{0}}{R_{11}}, \Longrightarrow|\vec{p}|=\frac{N_{0}}{R_{11}}\left(1+v^{2}\right)^{1 / 2} .
$$

This gives

$$
|\vec{p}| \frac{d p^{i}}{d t}=-\frac{Q_{2} x_{3}}{r^{8}}\left[2|\vec{p}|^{2}+p_{3}^{2}-2\left|p_{11}\right|^{2}\right]=-\frac{3 Q_{2} x_{3}}{r^{8}}\left(p_{3}\right)^{2} .
$$

So

$$
\frac{\partial V}{\partial x_{3}}=\frac{3 Q_{2} x_{3} v^{2} N_{0}}{r^{8} R_{11}} \Longrightarrow V(r)=-\frac{Q_{2} N_{0} v^{2}}{2 r^{6} R_{11}}
$$

which corresponds to a phase shift of

$$
\delta=\frac{3 \pi v Q_{2} N_{0}}{16 R_{11} b^{5}}=\frac{3(2 \pi)^{3} v N_{2} N_{0} l_{p}^{6}}{2 b^{5} R_{11}}
$$


To compare with the phase shift to be calculated in the gauge theory, we need to rescale

$$
b \rightarrow b l_{p}(2 \pi)^{1 / 3}, \quad v \rightarrow v \frac{R_{11}}{l_{p}}(2 \pi)^{-1 / 3} .
$$

(This is the rescaling that takes one from the 0-brane Lagrangian in 11 dimensional units to that used in Section 4.) This gives

$$
\delta=\frac{3 \pi v N_{2} N_{0}}{2 b^{5}}
$$

\subsection{Comments on scattering off nontrivial fixed points}

The phenomena that we have studied so far are just effective charges (except $\mathbb{R}^{9} / \mathbb{Z}_{2}^{\prime}$ ). There are more complicated orbifolds where extra degrees of freedom appear at the fixed points. This is the case of $\mathbb{R}^{4} / \mathbb{Z}_{2}$ [42] where the IR theory at the orbifold has 3 vector multiplets for the $W^{0}$ and $W^{ \pm}$bosons. An even more interesting example is $\mathbb{R}^{6} / \mathbb{Z}_{3}$ where the low-energy theory is a non-trivial interacting fixed point.

Whenever we have such extra degrees of freedom, there are nontrivial correlators of the energy-momentum tensor

$$
\left\langle T_{\mu_{1} \nu_{1}}\left(x_{1}\right) T_{\mu_{2} \nu_{2}}\left(x_{2}\right) \cdots T_{\mu_{n} \nu_{n}}\left(x_{n}\right)\right\rangle .
$$

where $x_{i}$ are localized at the fixed point. The lowest order effect will be an addition of

$$
\int d^{l} q K_{i j k l}(q) h_{i j}(-q, 0) h_{k l}(q, 0),
$$

to the action, where

$$
\begin{aligned}
g_{\mu \nu} & =\eta_{\mu \nu}+\int e^{i q_{\|} \cdot x_{\|}+i q_{\perp} \cdot x_{\perp}} h_{\mu \nu}\left(q_{\|}, q_{\perp}\right), \\
K_{i j k l}\left(q_{\|}\right) & =\left\langle T_{i j}\left(q_{\|}\right) T_{k l}\left(-q_{\|}\right)\right\rangle,
\end{aligned}
$$

Here $p_{\|}$is the momentum in the homogeneous directions $\left(6+1\right.$ directions for $\mathbb{R}^{4} / \mathbb{Z}_{2}$ and $4+1$ for $\left.\mathbb{R}^{6} / \mathbb{Z}_{3}\right)$. To lowest order the amplitude doesn't depend on the perpendicular momentum $p_{\perp}$ (in the 4 directions for $\mathbb{R}^{4} / \mathbb{Z}_{2}$ and 6 directions for $\mathbb{R}^{6} / \mathbb{Z}_{3}$ ) because $T_{\mu \nu}(x)$ contains a $\delta$-function $\delta\left(x_{\perp}\right)$. By conformal invariance we expect

$$
\begin{aligned}
K_{i j k l}(q) & =\frac{c}{q^{4}}\left\{\left(\delta_{i k} \delta_{j l}+\delta_{i l} \delta_{j k}-\frac{2}{D} \delta_{i j} \delta_{k l}\right) q^{4}\right. \\
& -q^{2}\left(q_{i} q_{k} \delta_{j l}+q_{i} q_{l} \delta_{j k}+q_{j} q_{l} \delta_{i k}+q_{j} q_{k} \delta_{j l}\right) \\
& \left.+\frac{2}{D} q^{2}\left(q_{i} q_{j} \delta_{k l}+q_{l} q_{k} \delta_{i j}\right)+\left(2-\frac{2}{D}\right) q_{i} q_{j} q_{k} q_{l}\right\}
\end{aligned}
$$

where $c$ is a generalization of the central charge.

Nevertheless, (2.4) cannot be used to calculate scattering of a graviton off the fixed point. This is because (2.4) contains $\delta\left(x_{\|}\right)$and to solve for a graviton plain wave one needs an extra information about the "profile" of the $\delta$-function (in analogy to scattering off a $\delta$-function potential in quantum-mechanics). 


\section{Behaviour of multi-loop diagrams}

The cases discussed in the previous section were all tree-level effects in supergravity. In contrast, the scattering off $\mathbb{R}^{8} / \mathbb{Z}_{2}$, or the effective membrane charge, can be deduced from a tadpole in a 1-loop diagram in type-IIA string theory [29]. Closed string tree-level in string theory is related to 1-loop in $\mathrm{M}$ (atrix)-theory, and 1-loop in string theory should be related to 2-loop in M(atrix)-theory.

In the next sections we will explicitly perform the calculation. In this section we wish to analyze, on general grounds, the behaviour of multi-loop diagrams.

M theory in eleven dimensions has no free parameters, once we have set the Planck scale to 1. Perturbation theory is possible because we are looking at low energy, long distance scattering. In the DKPS formalism [46] this corresponds to large impact parameter $b$ and low velocity $v$. Similarly, the BFSS Hamiltonian, after an appropriate rescaling of time, has no coupling constants. These facts indicate that the loop expansion we are doing is really an expansion depending on the physical parameters of the problem. To be precise, we show that $L$-loop diagrams behave as

$$
\frac{1}{b^{3(L-1)}} f_{L}\left(\frac{v}{b^{2}}\right) .
$$

The same argument will apply to the non-compact orbifolds we are considering, since there are no extra compactification parameters.

We write down the zero brane quantum mechanics Lagrangian :

$$
\begin{aligned}
& S=\frac{\left(\alpha^{\prime}\right)^{2}}{g \sqrt{\alpha^{\prime}}} \int d \tau\left[\frac{1}{2}\left(D_{\tau} \Phi_{I}\right)^{2}+\frac{1}{4}\left[\Phi_{I}, \Phi_{J}\right]^{2}+i \Psi^{T} D_{\tau} \Psi-\Psi^{T} \Gamma^{I}\left[\Phi_{I}, \Psi\right]\right] \\
& S=\frac{1}{R^{3}} \int d \tau\left[\frac{1}{2}\left(D_{\tau} \Phi_{I}\right)^{2}+\frac{1}{4}\left[\Phi_{I}, \Phi_{J}\right]^{2}+i \Psi^{T} D_{\tau} \Psi-\Psi^{T} \Gamma^{I}\left[\Phi_{I}, \Psi\right]\right]
\end{aligned}
$$

In going to the second line we used

$$
\alpha^{\prime}=\frac{l_{p}^{3}}{R}, \quad g \sqrt{\alpha^{\prime}}=R
$$

where $R$ is the eleven dimensional radius and we set $l_{p}=1$. Performing perturbation theory in this language weights a diagram with $L$ loops by a factor $R^{3(L-1)}$, since $R^{3}$ plays the role of Planck's constant. There is a rescaling of field variables and time which gets rid of the $R$ dependence completely [49,50]. Indeed, define

$$
\begin{aligned}
X & =R^{-1} \Phi \\
\tau & =\frac{t}{R} \\
\Theta & =R^{-3 / 2} \Psi
\end{aligned}
$$


In terms of these new variables the Lagrangian is

$$
S=\int d t\left[\frac{1}{2}\left(D_{t} X_{I}\right)^{2}+\frac{1}{4}\left[X_{I}, X_{J}\right]^{2}+i \Theta^{T} D_{t} \Theta-\Theta^{T} \Gamma^{I}\left[X_{I}, \Theta\right]\right]
$$

Now we will expand around the backgrounds

$$
\begin{aligned}
& x_{2}=v t \\
& x_{3}=b .
\end{aligned}
$$

The above rescalings allow us to relate background variables

$$
\begin{aligned}
\phi_{2} & =\widetilde{v} \tau \\
\phi_{b} & =\widetilde{b}
\end{aligned}
$$

to those in the $x$ variables.

$$
\begin{aligned}
& \widetilde{b}=R v \\
& \widetilde{v}=R^{2} v
\end{aligned}
$$

Let $\delta^{(L)}(b, v)$ be the phase shift computed with the Lagrangian (3.4) with backgrounds (3.5), and $\left(\delta^{\prime}\right)^{(L)}(b, v)$ be the phase shift computed with (3.1) and the backgrounds (3.6). They are related as

$$
\delta^{(L)}(b, v)=R^{3(L-1)}\left(\delta^{\prime}\right)^{(L)}(\widetilde{b}, \widetilde{v})
$$

This is solved by a phase shift of the form

$$
\delta^{(L)}(b, v)=b^{-3(L-1)} f_{L}\left(\frac{v}{b^{2}}\right)
$$

So the one loop answers are purely functions of $\frac{v}{b^{2}}$.

This argument shows that higher loops cannot give a correction to the $\frac{v^{3}}{b^{6}}$ term of the phase shift in the scattering of two zero branes. It allows terms proportional to $v^{3}$ but necessarily suppressed by higher powers of $b$ than $b^{6}$. Non perturbative effects will give factors $e^{-b^{3}}$, hence will not affect the coefficient of the $\frac{v^{3}}{b^{6}}$. Similarly, it is clear that we cannot get corrections to the $\frac{v}{b^{5}}$ term by higher than two-loop calculations.

The same argument can be generalized to the case of the $U(N)$ theory in the original BFSS proposal $\left(U(N) \times U(N)\right.$ for the $\mathbb{R}^{8} / \mathbb{Z}_{2}$ case). Large $\mathrm{N}$ counting shows that the $L$-loop diagrams behave, at leading order in large $N$, as

$$
\frac{N^{L+1}}{b^{3(L-1)}} f_{L}\left(\frac{v}{b^{2}}\right)
$$

This is the large $N$ generalization of (3.9). There are also diagrams with subleading dependence $\left(\sim N^{L+1-\rho}\right)$ on $N$. This is discussed in more detail in section (7). 


\section{Technical details}

In this section we will describe in detail the two-loop calculation.

\subsection{The M(atrix)-Model for $\mathbb{R}^{8} / \mathbb{Z}_{2}$}

Following [51,7] we obtain the $\mathrm{M}\left(\right.$ atrix)-Model for $\mathbb{R}^{8} / \mathbb{Z}_{2}$ by starting with a $U(2 N)$ SYM (3.4), picking the $U(2 N)$ matrix:

$$
U=\left(\begin{array}{cc}
\mathbf{I}_{N \times N} & 0 \\
0 & -\mathbf{I}_{N \times N}
\end{array}\right)
$$

and leaving only those modes which satisfy

$$
\begin{aligned}
\widetilde{A}_{0} & =U^{-1} \widetilde{A}_{0} U, \\
\widetilde{X}_{1} & =U^{-1} \widetilde{X}_{1} U, \\
\widetilde{X}_{I} & =-U^{-1} \widetilde{X}_{I} U, \quad I=2 \ldots 9, \\
\widetilde{\Psi} & =\Gamma^{2} \ldots \Gamma^{9} U^{-1} \widetilde{\Psi} U .
\end{aligned}
$$

We obtain a $U(N) \times U(N)$ gauge group, one bosonic field $X_{1}$ in the adjoint, and 8 bosonic fields $X_{2} \ldots X_{9}$ in the $(\mathbf{N}, \overline{\mathbf{N}})$. In terms of these the original $U(2 N)$ fields are

$$
\begin{aligned}
\widetilde{A}_{0} & =\left(\begin{array}{cc}
A_{0} & 0 \\
0 & A_{0}^{\prime}
\end{array}\right), \\
\widetilde{X}_{1} & =\left(\begin{array}{cc}
X_{1} & 0 \\
0 & X_{1}^{\prime}
\end{array}\right), \\
\widetilde{X}_{a} & =\left(\begin{array}{cc}
0 & X_{a} \\
X_{a}^{\dagger} & 0
\end{array}\right), \quad a=2 \ldots 9,
\end{aligned}
$$

We will denote the two $U(N)$ gauge fields by $A_{0}, A_{0}^{\prime}$ and the two bosonic fields corresponding to the two $U(N)$-s by $X_{1}, X_{1}^{\prime}$. The quotienting prescription (4.1) leaves 8 fermions in the $\mathbf{8}_{s}$ of $S O(8)$ and in the adjoint of $U(N) \times U(N)$ and additional fermions $\psi^{\dot{\alpha}}$ in the $\boldsymbol{8}_{c}$ of $S O(8)$ and in the $(\mathbf{N}, \overline{\mathbf{N}})$ of $U(N) \times U(N)$.

The explicit calculations will be carried out for $U(1) \times U(1)$. The bosonic background will be taken to be:

$$
\begin{aligned}
& X_{2}=v t, \\
& X_{3}=b .
\end{aligned}
$$

We decompose

$$
\psi^{\dot{\alpha}}=\chi^{\dot{\alpha}}+i \rho^{\dot{\alpha}},
$$


where $\chi$ and $\rho$ are real. We group them as follows:

$$
\xi^{i}=\left(\begin{array}{c}
\psi^{i^{\prime}}+\psi^{i} \\
2 \bar{\chi}^{i}
\end{array}\right), \quad \eta^{i}=\left(\begin{array}{c}
\psi^{i^{\prime}}-\psi^{i} \\
2 \bar{\rho}^{i}
\end{array}\right)
$$

We also define the bosonic fields $\Phi_{ \pm}$according to

$$
\operatorname{Im} X_{2}=\frac{\Phi_{+}-\Phi_{-}}{\sqrt{2}}, \quad A_{0}-A_{0}^{\prime}=\sqrt{2}\left(\Phi_{+}+\Phi_{-}\right)
$$

The Lagrangian is now a sum of bosonic, fermionic and ghost terms

$$
L=L_{b}+L_{f}+L_{g}
$$

The bosonic terms are

$$
L_{b}=L_{b}^{(2)}+L_{b}^{(3)}+L_{b}^{(4)}
$$

with the quadratic terms:

$$
\begin{aligned}
L_{b}^{(2)} & =\left(\partial_{t} \operatorname{Re} X_{a}\right)^{2}+\left(\partial_{t} \operatorname{Re} X_{2}\right)^{2}+\left(\partial_{t} \operatorname{Re} X_{3}\right)^{2} \\
& +\frac{1}{4}\left[\partial_{t}\left(X_{1}+X_{1}^{\prime}\right)\right]^{2}+\frac{1}{4}\left[\partial_{t}\left(A_{0}+A_{0}^{\prime}\right)\right]^{2} \\
& +\left(\partial_{t} \operatorname{Im} X_{a}\right)^{2}-4\left(b^{2}+v^{2} t^{2}\right)\left(\operatorname{Im} X_{a}\right)^{2}+\left(\partial_{t} \operatorname{Im} X_{3}\right)^{2}-4\left(b^{2}+v^{2} t^{2}\right)\left(\operatorname{Im} X_{3}\right)^{2} \\
& +\left(\partial_{t} \Phi_{+}\right)^{2}-4\left(b^{2}+v^{2} t^{2}+v\right) \Phi_{+}^{2}+\left(\partial_{t} \Phi_{-}\right)^{2}-4\left(b^{2}+v^{2} t^{2}-v\right) \Phi_{-}^{2} \\
& +\frac{1}{4}\left[\partial_{t}\left(X_{1}-X_{1}^{\prime}\right)\right]^{2}-\left(b^{2}+v^{2} t^{2}\right)\left(X_{1}-X_{1}^{\prime}\right)^{2}
\end{aligned}
$$

The cubic and quartic terms $L_{b}^{(3)}, L_{b}^{(4)}$ appear in the appendix. The fermionic quadratic terms are

$$
L_{f}=i \xi_{i}^{\dagger} \partial_{t} \xi^{i}+i \eta_{i}^{\dagger} \partial_{t} \eta^{i}+2 v t \eta_{i}^{\dagger} \sigma^{2} \eta^{i}+2 b \eta_{i}^{\dagger} \sigma^{1} \eta^{i}
$$

The cubic terms with fermions also appear in the appendix.

\subsection{One-loop}

The one-loop contribution to the phase-shift is given by:

$$
\delta(b, v)=\int \frac{d s}{s} \times \frac{1}{2 \sinh 2 s v} e^{-4 b^{2} s}[6+2 \cosh 4 s v-8 \cosh 2 s v]=\frac{v^{3}}{16 b^{6}}+\mathcal{O}\left(\frac{v^{5}}{b^{10}}\right),
$$

similarly to 46 . 


\subsection{Diagrams and propagators}

The propagators of the bosonic and fermionic fields can be read off from the kinetic operators in (4.5) and (4.6) (analytically continued to a Euclidean metric):

$$
\not D \equiv i \sigma^{3} \frac{d}{d t}-2 v t \sigma^{1}+2 b \sigma^{2}, \quad H \equiv-\frac{d^{2}}{d t^{2}}+4 v^{2} t^{2}+4 b^{2} .
$$

we find

$$
\not D^{2}=-\frac{d^{2}}{d t^{2}}+4 v^{2} t^{2}+4 b^{2}+2 v \sigma^{2}
$$

We also have the zero-mode operators

$$
\not D_{0} \equiv i \sigma^{3} \frac{d}{d t}, \quad H_{0} \equiv-\frac{d^{2}}{d t^{2}} .
$$

The propagators are defined as

$$
G(x, y)=\left\langle y\left|H^{-1}\right| x\right\rangle, \quad S(x, y)=-i\left\langle y\left|\not D^{-1}\right| x\right\rangle \sigma^{3},
$$

We find

$$
\begin{aligned}
G(x, y) & =\pi^{-1 / 2} \int d s e^{-4 b^{2} s} \sqrt{\frac{2 v}{2 \sinh 4 s v}} e^{-\frac{1}{2} v(x-y)^{2} \operatorname{coth} 2 s v-\frac{1}{2} v(x+y)^{2} \tanh 2 s v} \\
S(x, y) & =\pi^{-1 / 2} \int d s e^{-4 b^{2} s} \sqrt{\frac{2 v}{2 \sinh 4 s v}} e^{-\frac{1}{2} v(x-y)^{2} \operatorname{coth} 2 s v-\frac{1}{2} v(x+y)^{2} \tanh 2 s v} \\
& \times\left[\frac{v}{\sinh 2 s v}(x-y) \mathbf{I}+\frac{v}{\cosh 2 s v}(x+y) \sigma^{2}+2 b \sigma^{1} \cosh 2 s v+2 i b \sigma^{3} \sinh 2 s v\right]
\end{aligned}
$$

Finally, we define the propagators

$$
G_{ \pm}=\left\langle y\left|(H \pm 4 v)^{-1}\right| x\right\rangle, \quad G_{0}(x, y)=\left\langle y\left|H_{0}^{-1}\right| x\right\rangle, \quad S_{0}(x, y)=-i\left\langle y\left|\not D_{0}^{-1}\right| x\right\rangle \sigma^{3}
$$

and find

$$
\begin{aligned}
G_{0}(x, y) & =\frac{1}{2}|x-y|, \\
S_{0}(x, y) & =\frac{1}{2}(\theta(x-y)-\theta(y-x)) \mathbf{I} .
\end{aligned}
$$

The cubic and quartic interaction vertices give rise to the following kinds of diagrams:

1. Two cubic vertices joined by three bosonic propagators.

2. Two cubic vertices joined by one bosonic and two fermionic propagators.

3. Two cubic vertices joined by one bosonic and two ghost propagators.

4. A "figure-of-eight" bosonic diagram with a single quartic vertex. We will evaluate them in section (6). 


\subsection{Diagrams with tadpoles}

The zeroth order approximation of [46] was taking the D0-branes to move in a straight line. At the order of $L$-loops, one will in general find a correction to the classical trajectory. Thus, the $(L+1)$-th order calculation should start by substituting the $L$-th order corrected classical trajectory. This is tantamount to saying that the $L$-th order tadpoles cancel and thus we should only include 1PI diagrams if we expand around the corrected trajectory. Note that the corrected trajectory can differ by a large distance from the 0 -th order one for times large enough. In our case, the 1-loop effective potential vanishes up to order $\mathcal{O}\left(v^{3}\right)$, so we can just as well keep only the 1PI diagrams at 2-loop order.

\section{Wave-function contribution}

At the order of two-loops the "profile" of the bound-state wave-function could also contribute. For simplicity, we will restrict our discussion to the original BFSS model for flat $\mathbb{R}^{11}$. To see how the contribution arises, let us write down our Hamiltonian for $U(2 N)$ as

$$
\mathcal{H}=\mathcal{H}_{0}+\mathcal{H}_{\mathrm{SU}(\mathrm{N})}+\mathcal{H}_{\mathrm{SU}(\mathrm{N})}^{\prime}+V_{0}+U
$$

where $\mathcal{H}_{0}+V_{0}$ is the piece containing only the $U(1)$-parts of the two $U(N)$-s (i.e. the collective coordinates). $\mathcal{H}_{0}$ is the quadratic part that we used in our definition of the propagators $S(x, y)$ and $G(x, y)$ and $V_{0}$ are the interactions. $\mathcal{H}_{\mathrm{SU}(\mathrm{N})}$ is the $S U(N)$ part of the Hamiltonian, which has 16 supersymmetries and is identical to the BFSS Hamiltonian. $\mathcal{H}_{\mathrm{SU}(\mathrm{N})}^{\prime}$ is the $S U(N)$ Hamiltonian for the other $S U(N)$ corresponding to the second D0-brane bound state. Finally, $U$ consists of the off-diagonal interactions which contain interactions between the $(N, \bar{N})$ fields and the $S U(N)$ variables. In all our previous calculations we neglected $U$, and thus, the pieces $\mathcal{H}_{\mathrm{SU}(\mathrm{N})}+\mathcal{H}_{\mathrm{SU}(\mathrm{N})}^{\prime}$ decoupled.

Let us see how $U$ contributes. Let $X_{\mu}, X_{\mu}^{\prime}$ be the $U(N)$ coordinates. we defined

$$
X_{\mu}=\widehat{X}_{\mu}+x_{\mu} \mathbf{I}, \quad X_{\mu}^{\prime}=\widehat{X}_{\mu}^{\prime}+x_{\mu}^{\prime} \mathbf{I}
$$

where $\operatorname{tr}\left\{\widehat{X}_{\mu}\right\}=0$. Let $\psi_{\nu}$ denote the off-diagonal $(N, \bar{N})$ fermions. Then, $U$ contains terms like:

$$
U=\bar{\psi} \widehat{X} \psi+\cdots
$$

Such a term will give in two-loops an expression like

$$
\langle\bar{\psi} \psi \bar{\psi} \psi\rangle\left\langle\Psi_{0}|\widehat{X} \widehat{X}| \Psi_{0}\right\rangle
$$


(plus a bosonic contribution) where $\left|\Psi_{0}\right\rangle$ is the ground-state wave-function of $S U(N)$.

This will contribute

$$
\int d x d y\left(S(x, y) S(x, y)^{\dagger}+\partial_{y} G(x, y) \partial_{x} G(x, y)+\cdots\right) \mathcal{G}(x-y)
$$

where

$$
\delta_{a b} \mathcal{G}\left(t^{\prime}-t\right) \equiv\left\langle\Psi_{0}\left|T \widehat{X}_{a}\left(t^{\prime}\right) \widehat{X}_{b}(t)\right| \Psi_{0}\right\rangle
$$

We claim that for the order in which we are interested, the contribution of $\mathcal{G}(x-y)$ is determined by the commutation relations. The reason is that $S(x, y)$ in (5.1) localizes the $x-y$ variable to the vicinity of $(x-y) \sim(1 / b) \ll 1$. Thus, we may expand

$$
\begin{aligned}
\left\langle\Psi_{0}\left|T \widehat{X}_{a}\left(t^{\prime}\right) \widehat{X}_{b}(t)\right| \Psi_{0}\right\rangle & =\left\langle\Psi_{0}\left|\widehat{X}_{a} \widehat{X}_{b}\right| \Psi_{0}\right\rangle+\frac{1}{2}\left\langle\Psi_{0}\left|\left[\frac{d \widehat{X}_{a}}{d t}, \widehat{X}_{b}\right]\right| \Psi_{0}\right\rangle\left|t^{\prime}-t\right|+O\left(\left|t^{\prime}-t\right|^{2}\right) \\
& =\delta_{a b}\left(C+\frac{1}{2} i\left|t^{\prime}-t\right|+O\left(\left|t^{\prime}-t\right|^{2}\right)\right)
\end{aligned}
$$

(We assume here that $\left\langle\operatorname{tr} \widehat{X}^{2}\right\rangle$ is finite in the bound state, otherwise the behaviour as a function of $t-t^{\prime}$ might be more singular.) We do not know the coefficient $C$ which is related to the "size" of the bound-state, but when we plug this back to (5.1) the total contribution of $C$ will vanish because the bosonic diagrams will cancel the fermionic ones. This is as expected, since $C$ will give a contribution which is larger by a factor of $b$ than the rest of the diagrams. The remaining contribution of $\left|t^{\prime}-t\right|$, which was determined solely from the commutation relations, is the same as the free propagator and joins the diagrams with a free propagator, that we encountered before in such a way that the diagram will scale as $N^{3}$ like all the other diagrams that contribute.

\section{Evaluating the diagrams}

The diagrams with two cubic bosonic vertices give (the $J_{k}$ integrals are define in the appendix):

$$
\begin{aligned}
\Delta_{\text {bosonic }}^{(3-3)} & =80 J_{1}+8 J_{3}+2 J_{2}+64 J_{4}+14 J_{5}+2 J_{6}+4 J_{7}-\frac{1}{2} J_{8}-7 J_{9}+\frac{7}{2} J_{11}+\frac{1}{4} J_{13} \\
& +\frac{7}{2} J_{14}+\frac{9}{8} J_{15}+14 J_{16}-14 J_{17}+3 J_{18}+2 J_{20} \\
& =-\frac{47 \pi}{64 b v}-\frac{1415 \pi v}{8192 b^{5}} .
\end{aligned}
$$


The diagrams with one quartic bosonic vertex give (with the definitions of appendix B):

$$
\begin{aligned}
\Delta_{\text {bosonic }}^{(4)} & =7 K_{1}+4\left(K_{2}^{(+)}+K_{2}^{(-)}\right)+\frac{3}{4}\left(K_{3}^{(+)}+K_{3}^{(-)}\right)-\frac{1}{2} K_{4} \\
& =+\frac{\pi}{b v}+\frac{37 \pi v}{512 b^{5}}
\end{aligned}
$$

The ghost diagrams give:

$$
\Delta_{\text {ghosts }}^{(3-3)}=-4\left(J_{1}+J_{4}\right)=-\frac{\pi}{64 b v}+\frac{17 \pi v}{8192 b^{5}},
$$

Thus in total the "bosonic" contribution is

$$
\Delta_{\text {bosonic }}=\Delta_{\text {bosonic }}^{(3-3)}+\Delta_{\text {bosonic }}^{(4)}+\Delta_{\text {ghosts }}^{(3-3)}=\frac{\pi}{4 b v}-\frac{403 \pi v}{4096 b^{5}}
$$

The fermionic diagrams give (the $I$-s are defined in appendix B as well):

$$
\begin{aligned}
\Delta_{\text {fermionic }} & =-12 I_{2}-\left(I_{6}^{(+)}+I_{6}^{(-)}\right)-2 I_{4}+12 I_{5}-2 I_{7}-\left(I_{8}^{(+)}+I_{8}^{(-)}\right)-4 I_{3}-4 I_{1} \\
& =-\frac{\pi}{4 b v}+\frac{25 \pi v}{256 b^{5}} .
\end{aligned}
$$

Altogether we find the phase shift:

$$
\Delta=\Delta_{\text {fermionic }}+\Delta_{\text {bosonic }}=-\frac{3 \pi v}{4096 b^{5}} .
$$

As expected the terms proportional to $\frac{\pi}{b v}$ have cancelled.

\section{Discussion}

We have found that the $0+1 \mathrm{D} U(N) \times U(N)$ Quantum mechanical model for $\mathbb{R}^{8} / \mathbb{Z}_{2}$ predicts the leading phase shift to be

$$
\delta_{\text {Matrix }}(b, v)=-\frac{3 \pi N^{3} v}{4096 b^{5}}
$$

for the scattering of a bound state of $N$ partons off the orbifold point (using (3.10)). This result was obtained at two loop order of perturbation theory.

Our system is special in that it exhibits both a non-vanishing 1-loop contribution which behaves at leading order as $\frac{N^{2} v^{3}}{b^{6}}$ and a non-vanishing 2-loop contribution which behaves at leading order as $\frac{N^{3} v}{b^{5}}(3.10)$. The 1-loop contribution follows from a 0-brane interacting with its $\mathbb{Z}_{2}$ image to give a $\frac{N^{2} v^{4}}{b^{7}}$ potential. It is intriguing that the two-loop contribution is the dominant one for small $v$ and large $b$ even at finite $N$. 
On the other hand, supergravity predicts a phase shift of (for a charge $N_{2}=-2 \times \frac{1}{16}$ in the covering space in $(2.3)$ )

$$
\delta_{\text {SUGRA }}(b, v)=-\frac{3 \pi N v}{16 b^{5}}
$$

Thus we seem to be off by a factor of $\frac{N^{2}}{256}$. It is curious that we get the correct answer for $N=16$, which might have an interpretation in the context of [52].

This is perhaps the simplest example of a discrepancy in a system with 8 supersymmetries. Such systems have already posed problems in the past [32]. In that case the problem arises only when one blows up the orbifold point. Our example is singular in that it cannot be blown-up to a smooth CY manifold. From this point of view, perhaps it is not all that surprising to find a discrepancy. Trying to fix this problem promises to teach us something new about how to compactify M(atrix)-theory on curved manifolds.

Does this mean that the model for $\mathbb{R}^{8} / \mathbb{Z}_{2}$ is incorrect? It seems very hard to add more sectors to the $0+1 \mathrm{D}$ quantum mechanics to correct this result. We point out that the $0+1 \mathrm{D}$ model can be obtained from a reduction of a chiral model in $1+1 \mathrm{D}$ with $(0,8)$ supersymmetry. According to [35] a theory with 1 multiplet containing the $U(N) \times U(N)$ gauge field and $X_{1}, X_{1}^{\prime}$ fields together with the $\boldsymbol{8}_{s}$ fermionic fields and 1 multiplet consisting of the 8 fields $X_{a}$ in the $(N, \bar{N})$ together with the $\boldsymbol{8}_{c}$ fermions - is free of anomaly.

Before concluding we would like to add a caveat. We have not proven that the our results survive when one takes $N \rightarrow \infty$ first while keeping $b$ and $P_{\perp}$ fixed. In principle, the perturbation parameter is $N / b^{3}$ and the behaviour of $\mathrm{M}$ (atrix)-theory could differ from what we have suggested.2

\section{Acknowledgments}

It is a pleasure to thank V. Balasubramanian, T. Banks, A. Hashimoto, P. Horava, G. Lifschytz, V. Periwal, S.-J. Rey, E. Silverstein, S. Sethi, S. Shenker, L. Susskind and O. Tafjord for very helpful conversations. We would also like to thank all the participants of the Princeton M(atrix) Journal Club for the discussions.

2 We are grateful to T. Banks for discussions on the issue of the large $N$ limit in $\mathrm{M}($ atrix)theory. 


\section{Appendix A: The full Lagrangian for $\mathbb{R}^{8} / \mathbb{Z}_{2}$.}

With the definitions:

$$
\operatorname{Im} X_{2}=\frac{\Phi_{+}-\Phi_{-}}{\sqrt{2}}, \quad A_{0}-A_{0}^{\prime}=\sqrt{2}\left(\Phi_{+}+\Phi_{-}\right),
$$

the bosonic quadratic terms in the Lagrangian are:

$$
\begin{aligned}
L_{b}^{(2)} & =\left(\partial_{t} \operatorname{Re} X_{a}\right)^{2}+\left(\partial_{t} \operatorname{Re} X_{2}\right)^{2}+\left(\partial_{t} \operatorname{Re} X_{3}\right)^{2} \\
& +\frac{1}{4}\left[\partial_{t}\left(X_{1}+X_{1}^{\prime}\right)\right]^{2}+\frac{1}{4}\left[\partial_{t}\left(A_{0}+A_{0}^{\prime}\right)\right]^{2} \\
& +\left(\partial_{t} \operatorname{Im} X_{a}\right)^{2}-4\left(b^{2}+v^{2} t^{2}\right)\left(\operatorname{Im} X_{a}\right)^{2}+\left(\partial_{t} \operatorname{Im} X_{3}\right)^{2}-4\left(b^{2}+v^{2} t^{2}\right)\left(\operatorname{Im} X_{3}\right)^{2} \\
& +\left(\partial_{t} \Phi_{+}\right)^{2}-4\left(b^{2}+v^{2} t^{2}+v\right) \Phi_{+}^{2}+\left(\partial_{t} \Phi_{-}\right)^{2}-4\left(b^{2}+v^{2} t^{2}-v\right) \Phi_{-}^{2} \\
& +\frac{1}{4}\left[\partial_{t}\left(X_{1}-X_{1}^{\prime}\right)\right]^{2}-\left(b^{2}+v^{2} t^{2}\right)\left(X_{1}-X_{1}^{\prime}\right)^{2}
\end{aligned}
$$

the cubic terms:

$$
\begin{aligned}
L_{b}^{(3)} & =8 b\left(\operatorname{Im} X_{a}\right)\left(\operatorname{Im} X_{3}\right)\left(\operatorname{Re} X_{a}\right)+8 b\left(\operatorname{Im} X_{2}\right)\left(\operatorname{Im} X_{3}\right)\left(\operatorname{Re} X_{2}\right)+2 b\left(A_{0}-A_{0}^{\prime}\right)^{2}\left(\operatorname{Re} X_{3}\right) \\
& -8 b\left(\operatorname{Im} X_{a}\right)^{2}\left(\operatorname{Re} X_{3}\right)-8 b\left(\operatorname{Im} X_{2}\right)^{2}\left(\operatorname{Re} X_{3}\right)+8 v t\left(\operatorname{Im} X_{a}\right)\left(\operatorname{Im} X_{2}\right)\left(\operatorname{Re} X_{a}\right) \\
& +2 v t\left(A_{0}-A_{0}^{\prime}\right)^{2}\left(\operatorname{Re} X_{2}\right)-8 v t\left(\operatorname{Im} X_{a}\right)^{2}\left(\operatorname{Re} X_{2}\right)-8 v t\left(\operatorname{Im} X_{3}\right)^{2}\left(\operatorname{Re} X_{2}\right) \\
& +8 v t\left(\operatorname{Im} X_{2}\right)\left(\operatorname{Im} X_{3}\right)\left(\operatorname{Re} X_{3}\right)-2 b\left(\operatorname{Re} X_{3}\right)\left(X_{1}-X_{1}^{\prime}\right)^{2}-2 v t\left(\operatorname{Re} X_{2}\right)\left(X_{1}-X_{1}^{\prime}\right)^{2} \\
& -2\left(A_{0}-A_{0}^{\prime}\right)\left(\operatorname{Re} X_{a}\right)\left(\partial_{t} \operatorname{Im} X_{a}\right)-2\left(A_{0}-A_{0}^{\prime}\right)\left(\operatorname{Re} X_{2}\right)\left(\partial_{t} \operatorname{Im} X_{2}\right) \\
& -2\left(A_{0}-A_{0}^{\prime}\right)\left(\operatorname{Re} X_{3}\right)\left(\partial_{t} \operatorname{Im} X_{3}\right)+2\left(A_{0}-A_{0}^{\prime}\right)\left(\operatorname{Im} X_{a}\right)\left(\partial_{t} \operatorname{Re} X_{a}\right) \\
& +2\left(A_{0}-A_{0}^{\prime}\right)\left(\operatorname{Im} X_{2}\right)\left(\partial_{t} \operatorname{Re} X_{2}\right)+2\left(A_{0}-A_{0}^{\prime}\right)\left(\operatorname{Im} X_{3}\right)\left(\partial_{t} \operatorname{Re} X_{3}\right)
\end{aligned}
$$

and the quartic terms

$$
\begin{aligned}
L_{b}^{(4)} & =\left(A_{0}-A_{0}^{\prime}\right)^{2}\left(\operatorname{Im} X_{a}\right)^{2}+\left(A_{0}-A_{0}^{\prime}\right)^{2}\left(\operatorname{Im} X_{2}\right)^{2}+\left(A_{0}-A_{0}^{\prime}\right)^{2}\left(\operatorname{Im} X_{3}\right)^{2} \\
& +\left(A_{0}-A_{0}^{\prime}\right)^{2}\left(\operatorname{Re} X_{a}\right)^{2}-2\left(\operatorname{Im} X_{b}\right)^{2}\left(\operatorname{Re} X_{a}\right)^{2}-4\left(\operatorname{Im} X_{2}\right)^{2}\left(\operatorname{Re} X_{a}\right)^{2} \\
& -4\left(\operatorname{Im} X_{3}\right)^{2}\left(\operatorname{Re} X_{a}\right)^{2}+4\left(\operatorname{Im} X_{a}\right)\left(\operatorname{Im} X_{b}\right)\left(\operatorname{Re} X_{a}\right)\left(\operatorname{Re} X_{b}\right)-2\left(\operatorname{Im} X_{a}\right)^{2}\left(\operatorname{Re} X_{b}\right)^{2} \\
& +8\left(\operatorname{Im} X_{a}\right)\left(\operatorname{Im} X_{2}\right)\left(\operatorname{Re} X_{a}\right)\left(\operatorname{Re} X_{2}\right)+\left(A_{0}-A_{0}^{\prime}\right)^{2}\left(\operatorname{Re} X_{2}\right)^{2}-4\left(\operatorname{Im} X_{a}\right)^{2}\left(\operatorname{Re} X_{2}\right)^{2} \\
& -4\left(\operatorname{Im} X_{3}\right)^{2}\left(\operatorname{Re} X_{2}\right)^{2}+8\left(\operatorname{Im} X_{a}\right)\left(\operatorname{Im} X_{3}\right)\left(\operatorname{Re} X_{a}\right)\left(\operatorname{Re} X_{3}\right) \\
& +8\left(\operatorname{Im} X_{2}\right)\left(\operatorname{Im} X_{3}\right)\left(\operatorname{Re} X_{2}\right)\left(\operatorname{Re} X_{3}\right)+\left(A_{0}-A_{0}^{\prime}\right)^{2}\left(\operatorname{Re} X_{3}\right)^{2} \\
& -4\left(\operatorname{Im} X_{a}\right)^{2}\left(\operatorname{Re} X_{3}\right)^{2}-4\left(\operatorname{Im} X_{2}\right)^{2}\left(\operatorname{Re} X_{3}\right)^{2}-\left(\operatorname{Im} X_{a}\right)^{2}\left(X_{1}-X_{1}^{\prime}\right)^{2} \\
& -\left(\operatorname{Im} X_{2}\right)^{2}\left(X_{1}-X_{1}^{\prime}\right)^{2}-\left(\operatorname{Im} X_{3}\right)^{2}\left(X_{1}-X_{1}^{\prime}\right)^{2} \\
& -\left(\operatorname{Re} X_{a}\right)^{2}\left(X_{1}-X_{1}^{\prime}\right)^{2}-\left(\operatorname{Re} X_{2}\right)^{2}\left(X_{1}-X_{1}^{\prime}\right)^{2} \\
& -\left(\operatorname{Re} X_{3}\right)^{2}\left(X_{1}-X_{1}^{\prime}\right)^{2}
\end{aligned}
$$


The fermionic variables appear in:

$$
\begin{aligned}
L_{f} & =i \xi_{i}^{\dagger} \partial_{t} \xi^{i}+i \eta_{i}^{\dagger} \partial_{t} \eta^{i}+2 v t \eta_{i}^{\dagger} \sigma^{2} \eta^{i}+2 b \eta_{i}^{\dagger} \sigma^{1} \eta^{i} \\
& -i\left(\operatorname{Re} X_{a}\right)\left\{\eta^{i T} \sigma^{1} \eta^{j} \gamma_{i j}^{a}+\bar{\eta}_{i}^{T} \sigma^{1} \bar{\eta}_{j} \gamma^{i j, a}\right\} \\
& +\frac{i}{\sqrt{2}}\left(\Phi_{-}-\Phi_{+}\right)\left\{\eta_{i}^{\dagger} \sigma^{+} \xi^{i}-\xi^{i^{\dagger}} \sigma^{-} \eta_{i}\right\}-\left(\operatorname{Im} X_{3}\right)\left\{\eta_{i}^{\dagger} \sigma^{+} \xi^{i}+\xi^{i^{\dagger}} \sigma^{-} \eta_{i}\right\} \\
& +i\left(\operatorname{Im} X_{a}\right)\left\{\eta^{i} \sigma^{+} \xi^{j} \gamma_{i j}^{a}+\xi_{i}^{\dagger} \sigma^{-} \bar{\eta}_{j} \gamma^{i j, a}\right\}+\frac{i}{2}\left(X^{1}-X^{1^{\prime}}\right)\left\{\xi_{i}^{\dagger}\left(\mathbf{I}-\sigma^{3}\right) \eta^{i}-\eta_{i}^{\dagger}\left(\mathbf{I}-\sigma^{3}\right) \xi^{i}\right\} \\
& +\frac{\sqrt{2}}{2} i\left(\Phi_{-}+\Phi_{+}\right)\left\{\xi_{i}^{\dagger}\left(\mathbf{I}-\sigma^{3}\right) \eta^{i}-\eta_{i}^{\dagger}\left(\mathbf{I}-\sigma^{3}\right) \xi^{i}\right\} \\
& +2\left(\operatorname{Re} X_{2}\right) \eta_{i}^{\dagger} \sigma^{2} \eta^{i}+2\left(\operatorname{Re} X_{3}\right) \eta_{i}^{\dagger} \sigma^{1} \eta^{i}
\end{aligned}
$$

\section{Appendix B: Integrals.}

The fields have the following propagators:

$$
\begin{aligned}
\operatorname{Re} X_{2} & \rightarrow \frac{1}{\sqrt{2}} G_{0}, \\
\operatorname{Im} X_{3} & \rightarrow \frac{1}{\sqrt{2}} G, \\
\operatorname{Re} X_{3} & \rightarrow \frac{1}{\sqrt{2}} G_{0}, \\
\operatorname{Im} X_{a} & \rightarrow \frac{1}{\sqrt{2}} G, \\
\operatorname{Re} X_{a} & \rightarrow \frac{1}{\sqrt{2}} G_{0}, \\
\Phi_{-} & \rightarrow \frac{1}{\sqrt{2}} G_{-}, \\
\Phi_{+} & \rightarrow \frac{1}{\sqrt{2}} G_{+}, \\
A_{0}+A_{0}^{\prime} & \rightarrow \sqrt{2} G_{0}, \\
X_{1}-X_{1}^{\prime} & \rightarrow \sqrt{2} G, \\
\chi & \rightarrow S_{0}, \\
\eta & \rightarrow S,
\end{aligned}
$$


We are going to need a few integrals. We calculated them with the definitions:

$$
\begin{aligned}
G(x, y) & =\pi^{-1 / 2} \int d s e^{-4 b^{2} s} \sqrt{\frac{2 v}{2 \sinh 4 s v}} e^{-\frac{1}{2} v(x-y)^{2} \operatorname{coth} 2 s v-\frac{1}{2} v(x+y)^{2} \tanh 2 s v} \\
S(x, y) & =\pi^{-1 / 2} \int d s e^{-4 b^{2} s} \sqrt{\frac{2 v}{2 \sinh 4 s v}} e^{-\frac{1}{2} v(x-y)^{2} \operatorname{coth} 2 s v-\frac{1}{2} v(x+y)^{2} \tanh 2 s v} \\
& \times\left[\frac{v}{\sinh 2 s v}(x-y) \mathbf{I}+\frac{v}{\cosh 2 s v}(x+y) \sigma^{2}+2 b \sigma^{1} \cosh 2 s v+2 i b \sigma^{3} \sinh 2 s v\right] \\
G_{0}(x, y) & =\frac{1}{2}|x-y| \\
S_{0}(x, y) & =\frac{1}{2}(\theta(x-y)-\theta(y-x)) \mathbf{I} \\
G_{ \pm}(x, y) & =\pi^{-1 / 2} \int d s e^{ \pm 4 v s-4 b^{2} s} \sqrt{\frac{2 v}{2 \sinh 4 s v}} e^{-\frac{1}{2} v(x-y)^{2} \operatorname{coth} 2 s v-\frac{1}{2} v(x+y)^{2} \tanh 2 s v} \\
S_{ \pm}(x, y) & =\pi^{-1 / 2} \int d s e^{ \pm 4 v s-4 b^{2} s} \sqrt{\frac{2 v}{2 \sinh 4 s v}} e^{-\frac{1}{2} v(x-y)^{2} \operatorname{coth} 2 s v-\frac{1}{2} v(x+y)^{2} \tanh 2 s v}
\end{aligned}
$$

For the bosonic diagrams with two cubic vertices we will need:

$$
\begin{gathered}
J_{1}=b^{2} \int d x d y G_{0} G G=\frac{\pi}{512 b v}-\frac{95 \pi v}{262144 b^{5}}+\mathcal{O}\left(v^{2}\right), \\
J_{2}=b^{2} \int d x d y G_{0} G\left(G_{+}+G_{-}\right)=\frac{\pi}{256 b v}+\frac{205 \pi v}{131072 b^{5}}+\mathcal{O}\left(v^{2}\right), \\
J_{3}=b^{2} \int d x d y G_{0}\left(G_{+} G_{+}+G_{-} G_{-}\right)=\frac{\pi}{256 b v}+\frac{865 \pi v}{131072 b^{5}}+\mathcal{O}\left(v^{2}\right), \\
J_{4}=v^{2} \int d x d y \quad x y G_{0} G G=\frac{\pi}{512 b v}-\frac{41 \pi v}{262144 b^{5}}+\mathcal{O}\left(v^{2}\right), \\
J_{5}=v^{2} \int d x d y x y G_{0} G\left(G_{+}+G_{-}\right)=\frac{\pi}{256 b v}+\frac{19 \pi v}{131072 b^{5}}+\mathcal{O}\left(v^{2}\right), \\
J_{6}=v^{2} \int d x d y x y G_{0}\left(G_{+} G_{+}+G_{-} G_{-}\right)=\frac{\pi}{256 b v}+\frac{151 \pi v}{131072 b^{5}}+\mathcal{O}\left(v^{2}\right), \\
J_{7}=v^{2} \int d x d y x y G_{0} G_{+} G_{-}=\frac{\pi}{512 b v}+\frac{7 \pi v}{262144 b^{5}}+\mathcal{O}\left(v^{2}\right), \\
J_{8}=\int d x d y G_{0} \partial_{x} G_{+} \partial_{y} G_{-}=-\frac{\pi}{64 b v}-\frac{17 \pi v}{16384 b^{5}}+\mathcal{O}\left(v^{2}\right), \\
J_{9}=\int d x d y\left(G_{+}+G_{-}\right) \partial_{y} G \partial_{x} G_{0}=\frac{\pi}{16 b v}+\frac{53 \pi v}{4096 b^{5}}+\mathcal{O}\left(v^{2}\right), \\
J_{10}=\int d x d y\left(G_{+} \partial_{x} G_{+}+G_{-} \partial_{x} G_{-}\right) \partial_{y} G_{0}=\frac{\pi}{16 b v}+\frac{89 \pi v}{4096 b^{5}}+\mathcal{O}\left(v^{2}\right),
\end{gathered}
$$




$$
\begin{aligned}
J_{11} & =\int d x d y G_{0}\left(G_{+}+G_{-}\right) \partial_{x} \partial_{y} G=-\frac{\pi}{32 b v}-\frac{83 \pi v}{8192 b^{5}}+\mathcal{O}\left(v^{2}\right), \\
J_{12} & =\int d x d y G_{0}\left(G_{+} \partial_{x} \partial_{y} G_{+}+G_{-} \partial_{x} \partial_{y} G_{-}\right)=-\frac{\pi}{32 b v}-\frac{89 \pi v}{8192 b^{5}}+\mathcal{O}\left(v^{2}\right), \\
J_{13} & =\int d x d y G_{0}\left(G_{-} \partial_{x} \partial_{y} G_{+}+G_{+} \partial_{x} \partial_{y} G_{-}\right)=-\frac{\pi}{32 b v}-\frac{65 \pi v}{8192 b^{5}}+\mathcal{O}\left(v^{2}\right), \\
J_{14} & =\int d x d y\left(G_{+}+G_{-}\right) G \partial_{x} \partial_{y} G_{0}=-\frac{\pi}{8 b v}-\frac{29 \pi v}{2048 b^{5}}+\mathcal{O}\left(v^{2}\right), \\
J_{15} & =\int d x d y\left(G_{+} G_{+}+G_{-} G_{-}\right) \partial_{x} \partial_{y} G_{0}=-\frac{\pi}{8 b v}-\frac{89 \pi v}{2048 b^{5}}+\mathcal{O}\left(v^{2}\right),
\end{aligned}
$$

$$
\begin{aligned}
J_{16} & =v \int d x d y x G_{0}\left(G_{+}-G_{-}\right) \partial_{y} G=0+\frac{21 \pi v}{16384 b^{5}}+\mathcal{O}\left(v^{2}\right), \\
J_{17} & =v \int d x d y y G\left(G_{+}-G_{-}\right) \partial_{x} G_{0}=0-\frac{3 \pi v}{2048 b^{5}}+\mathcal{O}\left(v^{2}\right), \\
J_{18} & =v \int d x d y y\left(G_{+} G_{+}-G_{-} G_{-}\right) \partial_{x} G_{0}=0-\frac{3 \pi v}{1024 b^{5}}+\mathcal{O}\left(v^{2}\right), \\
J_{19} & =v \int d x d y y G_{0}\left(G_{+} \partial_{x} G_{+}-G_{-} \partial_{x} G_{-}\right)=0+\frac{3 \pi v}{2048 b^{5}}+\mathcal{O}\left(v^{2}\right), \\
J_{20} & =v \int d x d y \text { y } G_{0}\left(G_{+} \partial_{x} G_{-}-G_{-} \partial_{x} G_{+}\right)=0+\frac{9 \pi v}{8192 b^{5}}+\mathcal{O}\left(v^{2}\right),
\end{aligned}
$$

For the "figure of eight" diagrams with one quartic vertex we will need:

$$
\begin{aligned}
K_{1} & =\int d x G(x, x) G(x, x) d x=\frac{\pi}{16 b v}-\frac{7 \pi v}{4096 b^{5}}+\mathcal{O}\left(v^{2}\right), \\
K_{2}^{( \pm)} & =\int d x G(x, x) G_{+}(x, x) d x= \pm \frac{\pi}{64 b^{3}}+\frac{\pi}{16 b v}+\frac{29 \pi v}{4096 b^{5}}+\mathcal{O}\left(v^{2}\right), \\
K_{3}^{( \pm)} & =\int d x G_{+}(x, x) G_{+}(x, x) d x= \pm \frac{\pi}{32 b^{3}}+\frac{\pi}{16 b v}+\frac{89 \pi v}{4096 b^{5}}+\mathcal{O}\left(v^{2}\right), \\
K_{4} & =\int d x G_{+}(x, x) G_{-}(x, x) d x=\frac{\pi}{16 b v}+\frac{41 \pi v}{4096 b^{5}}+\mathcal{O}\left(v^{2}\right) .
\end{aligned}
$$


and for the diagrams with two cubic fermionic vertices we need:

$$
\begin{aligned}
I_{1} & =\int d x d y \operatorname{tr}\left\{S(x, y) \sigma^{1} S(y, x) \sigma^{1}\right\} G_{0}(x, y)=-\frac{\pi}{32 b v}+\frac{125 \pi v}{16384 b^{5}}+\mathcal{O}\left(v^{2}\right), \\
I_{2} & =\int d x d y \operatorname{tr}\left\{S(x, y) \sigma^{1} S(y, x)^{*} \sigma^{1}\right\} G_{0}(x, y)=0, \\
I_{3} & =\int d x d y \operatorname{tr}\left\{S(x, y) \sigma^{2} S(y, x) \sigma^{2}\right\} G_{0}(x, y)=-\frac{\pi}{32 b v}+\frac{35 \pi v}{16384 b^{5}}+\mathcal{O}\left(v^{2}\right), \\
I_{4} & =\int d x d y \operatorname{tr}\left\{S_{0}(x, y) \sigma^{-} S(y, x) \sigma^{+}\right\} G(x, y)=-\frac{\pi}{8 b v}+\frac{5 \pi v}{1024 b^{5}}+\mathcal{O}\left(v^{2}\right), \\
I_{5} & =\int d x d y \operatorname{tr}\left\{S_{0}(x, y) \sigma^{-} S(y, x)^{*} \sigma^{+}\right\} G(x, y)=-\frac{\pi}{8 b v}+\frac{5 \pi v}{1024 b^{5}}+\mathcal{O}\left(v^{2}\right), \\
I_{6}^{( \pm)} & =\int d x d y \operatorname{tr}\left\{S_{0}(x, y) \sigma^{-} S(y, x) \sigma^{+}\right\} G_{ \pm}(x, y)=\mp \frac{3 \pi}{32 b^{3}}-\frac{\pi}{8 b v}-\frac{25 \pi v}{1024 b^{5}}+\mathcal{O}\left(v^{2}\right), \\
I_{7} & =\int d x d y \operatorname{tr}\left\{S_{0}(x, y)\left(\mathbf{I}-\sigma^{3}\right) S(y, x)\left(\mathbf{I}-\sigma^{3}\right)\right\} G(x, y)=-\frac{\pi}{8 b v}+\frac{5 \pi v}{1024 b^{5}}+\mathcal{O}\left(v^{2}\right), \\
I_{8}^{( \pm)} & =\int d x d y \operatorname{tr}\left\{S_{0}(x, y)\left(\mathbf{I}-\sigma^{3}\right) S(y, x)\left(\mathbf{I}-\sigma^{3}\right)\right\} G_{ \pm}(x, y) \\
& =\mp \frac{3 \pi}{32 b^{3}}-\frac{\pi}{8 b v}-\frac{25 \pi v}{1024 b^{5}}+\mathcal{O}\left(v^{2}\right) .
\end{aligned}
$$




\section{References}

[1] T. Banks, W. Fischler, S.H. Shenker and L. Susskind, "M Theory As A Matrix Model: A Conjecture," hep-th/9610043

[2] W. Taylor, "D-brane field theory on compact spaces," hep-th/9611042

[3] L. Susskind, "T Duality in M(atrix) Theory and S Duality in Field Theory," hepth/9611164

[4] O.J. Ganor, S. Ramgoolam and W. Taylor IV, "Branes, Fluxes and Duality in M(atrix)-Theory," hep-th/9611202

[5] U.H. Danielsson and G. Ferretti, "The Heterotic Life OF The D-Particle," hepth/9610082

[6] S. Kachru and E. Silverstein, "On Gauge Bosons in the Matrix Model Approach to M Theory," hep-th/9612162

[7] N. Kim and S.-J. Rey, "M(atrix) Theory on an Orbifold and Twisted Membrane," hep-th/9701139

[8] Z. Guralnik and S. Ramgoolam, "Torons and D-Brane Bound States," hep-th/9702099

[9] S. Sethi and L. Susskind, "Rotational Invariance in the M(atrix) Formulation of Type IIB Theory," hep-th/9702101

[10] D. Berenstein and R. Corrado, "M(atrix)-Theory in Various Dimensions," hepth/9702108

[11] M. Rozali, "Matrix Theory and U-duality in Seven Dimensions," hep-th/9702136

[12] W. Fischler, E. Halyo, A. Rajaraman, and L. Susskind, "The Incredible Shrinking Torus," hep-th/9703102

[13] T. Banks, N. Seiberg and S. Shenker, "Branes from Matrices," hep-th/9612157

[14] R. Gopakumar, "BPS States In Matrix Strings", hep-th/9704030

[15] D. Berenstein, R. Corrado, and J. Distler, "On the Moduli Spaces of M(atrix)-Theory Compactifications," hep-th/9704087

[16] M. Berkooz, M. Rozali and N. Seiberg, "Matrix Description of M-theory on $T^{4}$ and $T^{5}, "$ hep-th/9704089

[17] W. Fischler and A. Rajaraman, "M(atrix) String Theory on K3," hep-th/9704123

[18] T. Banks and N. Seiberg, "Strings from Matrices," hep-th/9702187

[19] L. Motl, "Proposals on Non-perturbative Superstring Interactions," hep-th/9701025

[20] R. Dijkgraaf, E. Verlinde, H. Verlinde, "Matrix String Theory," hep-th/9703030

[21] T. Banks and L. Motl, "Heterotic Strings from Matrices," hep-th/9703218

[22] D.A. Lowe, "Heterotic Matrix String Theory," hep-th/9704041

[23] S.-J. Rey, "Heterotic M(atrix) Strings and Their Interactions," hep-th/9704158

[24] Petr Horava, "Matrix Theory and Heterotic Strings on Tori", hep-th/9705055

[25] O. Aharony and M. Berkooz, "Membrane Dynamics in M(atrix) Theory," hepth/9611215 
[26] G. Lifschytz and S. Mathur, "Supersymmetry and Membrane Interactions in M(atrix) Theory," hep-th/9612087

[27] G. Lifschytz, "Four-Brane and Six-Brane Interactions in M(atrix) Theory," hepth/9612223

[28] J.Polchinski, P.Pouliot, "Membrane Scattering with M-Momentum Transfer," hepth/9704029

[29] E. Witten and C. Vafa, "A One-Loop Test Of String Duality," hep-th/9505053

[30] S. Sethi and M. Stern, "D-Brane Bound States Redux," hep-th/9705046

[31] M. R. Douglas, "Enhanced Gauge Symmetry in M(atrix) Theory," hep-th/9612126

[32] M. R. Douglas, H. Ooguri and S. H. Shenker, "Issues in (M)atrix Model Compactification," hep-th/9702203

[33] E. Witten, "Some Comments on String Dynamics," contributed to Strings '95, hepth/9507121

[34] M. Berkooz and M. Rozali, "String Dualities from Matrix Theory," hep-th/9705175

[35] T. Banks, N. Seiberg, and E. Silverstein, "Zero and One-dimensional Probes with $N=8$ Supersymmetry", hep-th/9703052

[36] M.R. Douglas, B.R. Greene and D.R. Morrison, "Orbifold Resolution By D-Branes", hep-th/9704151

[37] K. Becker and M. Becker, "A Two-Loop Test of M(atrix) Theory", hep-th/9705091

[38] N. Kim and S.-J. Rey, "M(atrix) Theory on $T_{5} / Z_{2}$ Orbifold and Five-Brane", hepth/9705132

[39] N. Kim and S.-J. Rey, to appear.

[40] K. Dasgupta and S. Mukhi, "Orbifolds of M-Theory," Nucl. Phys. B465 (399) 96, hepth/9512196

[41] E. Witten, "Five-Branes and M-Theory On an Orbifold," Nucl. Phys. B463 (96) 383, hep-th/9512219

[42] E. Witten, "String Theory Dynamics in Various Dimensions," Nucl. Phys. B443 (95) 85, hep-th/9503124

[43] K. Dasgupta and S. Mukhi, "A Note on Low-Dimensional String Compactifications," hep-th/9612188

[44] E. Witten, "Phase Transitions In M-Theory And F-Theory," hep-th/9603150

[45] A. Fayyazuddin and D.J. Smith, "A note on $T^{5} / Z_{2}$ compactification of the M-theory matrix model," hep-th/9703208

[46] M.R. Douglas, D. Kabat, P. Pouliot and S. Shenker, "D-branes and Short Distances in String Theory," hep-th/9608024

[47] C.Bachas, M. Porrati, "Pair Creation of Open Strings in an Electric Field," Phys. Lett. 296B (77) 92, hep-th/9209032

[48] J.G. Russo, "BPS Bound States, Supermembranes and T-Duality in M-Theory," hepth/9703118 
[49] D. Kabat and P. Pouliot, "A Comment on Zero Brane Quantum Mechanics", hepth/9603127, Phys. Rev. Lett.77 (96) 1004

[50] U.H. Danielsson, G. Ferretti and B. Sundborg, "D-Particle Dynamics and BoundStates", hep-th/9603081, Int. Jour. Mod. Phys. A11 (96) 5463

[51] M. Douglas and G. Moore, "D-branes, Quivers And ALE Instantons", hep-th/9603167

[52] L. Susskind, "Another Conjecture about M(atrix) Theory," hep-th/9704080 